\title{
Infection Control in Health Care Facilities
}

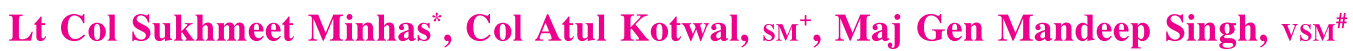

MJAFI 2011; $67: 7-8$

Key Words : Health care worker (HCW); Infection control; Occupational exposure

$\mathrm{I}^{\mathrm{n}}$ nfection control is an essential part of health care at all levels. In developing countries like India, more often than not it is not only under recognized and under reported but also an under supported aspect in health care facilities (HCFs). It is a well established fact that health care workers (HCWs) are at risk of infection with blood-borne pathogens. Infection control addresses factors related to the spread of infections within the health-care setting, including prevention, monitoring/ investigation of demonstrated or suspected spread of infection within a particular health-care setting, and management.

All HCFs and services must have a strategy in place to protect $\mathrm{HCW}$ s that operates in unison with a comprehensive infection prevention and control programme (IPCP). A comprehensive IPCP includes, but is not limited to, dedicated staff and appropriate organisation of the programme, technical guidelines, health care worker safety, surveillance of infections and monitoring of compliance with the programme, laboratory support and environmental controls. Such a programme must be in place and well established to be well prepared for any new infectious disease threat. The form of exposure most likely to result in occupational blood-borne virus (BBV) infection is a needlestick injury (NSI) or splashes of blood and body fluids (BBF). Approximately three million percutaneous exposures to blood-borne pathogens occur annually amongst 35 million HCWs worldwide. These injuries are estimated to result in 16,000 hepatitis C, 66,000 hepatitis B and 200-5,000 HIV infections. Over $90 \%$ of these infections are occurring in low-income countries, and most are preventable [1]. In this issue, Sangwan et al [2], report a high level of occupational exposure to $\mathrm{BBF}$ and consequent risk of infection amongst a group of HCWs in the Armed Forces. This highlights the urgent need for interventions to enhance the occupational safety of HCWs in the
Armed Forces hospitals.

The integrated infection control/ health care worker safety strategy includes practices to minimise direct unprotected exposure to potentially infected blood, body fluids or secretions or Standard Precautions. In addition or arguably included with this is the appropriate management of body fluid exposures and immunisation against hepatitis B and other infectious diseases [3].

Inadequate implementation of standard/ universal precautions guidelines not only endanger the HCWs but also may lead to increased risk of transmission of infections within the HCFs. Healthcare Associated Infections (HAIs) have a substantial impact on morbidity, mortality and health costs. At least 5 to $10 \%$ of patients admitted to acute care hospitals acquire an infection. Factors that promote HAIs include underlying diseases and decreased patient immunity, invasive diagnostic and therapeutic techniques, the widespread antimicrobial resistance, lack of infection control measures and environmental hygiene. HAIs are preventable and infection control programmes are cost-effective [4].

The risk for occupationally acquired infection is an unavoidable part of daily patient care [5]. Prospective studies of HCWs have estimated that the average risk for HIV transmission after a percutaneous exposure is approximately $0.3 \%$, the risk of $\mathrm{HBV}$ transmission is 6 to $30 \%$ and the risk of $\mathrm{HCV}$ transmission is approximately $1.8 \%$ [6].

In a study carried out among HCWs $(n=3,556)$ in a tertiary care set-up at Chandigarh, India [6] fifteen percent had received only one dose, $26 \%$ two, $59 \%$ three and $2.5 \%$ received the booster dose of the HBV vaccine. $\mathrm{HBsAg}$ and anti-HCV prevalence was found to be $1.7 \%$ and $0.87 \%$ respectively. In this as well as another study it was found that HCWs, despite the awareness on HBV and $\mathrm{HCV}$ infection were noncompliant for $\mathrm{HBV}$ vaccination [7]. Another study carried out among health

\footnotetext{
${ }^{*}$ Assistant Professor (Community Medicine) \& Technical Editor, MJAFI, +Professor, Department of Community Medicine, AFMC, Pune40; "Addl DGAFMS (Medical Research), O/o DGAFMS, Ministry of Defence, 'M' Block, New Delhi-01.

E-mail : dratulkotwal@gmail.com
} 
care workers in a developed country found that compliance with standard precautions was poor, especially for the use of protective clothes and safety material [8]. In the study by Sangwan et al [2] a high level of occupational exposure has been shown despite retrospective reporting of occupational exposures being subject to recall bias. The incidence density of BBF exposure and NSIs episodes was 537.14 and 228.57 per 100 person years, respectively. All components of personal protective equipment (PPE) were available as per only 34.29 percent. The findings suggest that $\mathrm{HCWs}$ in Armed Forces Hospitals understand the principles of universal precautions (UPs) much better than their own practices. The findings demonstrate a low-level of overall compliance with UPs amongst HCWs of Armed Forces Hospitals.

Further studies are needed to identify new infection control strategies to improve protection of health care workers and to enhance compliance with established approaches. As health care is being reformed, the risk for and cost of occupationally acquired infection must be considered [5]. There is a need to reinforce compliance with standard hygiene precautions which is crucial for medical and technical personnel working in the health care set up [8]. A sustained commitment to the occupational health of all HCWs will ensure maximum protection for $\mathrm{HCWs}$ and patients and the availability of PPE and optimal medical care for all who need it [6]. Besides, it is imperative to have in place the system that encourages the $\mathrm{HCW}$ to report occupational exposures as well as to use personal protective equipment. In a study by Sencan et al [9] it was observed that there appeared to be under-reporting of potentially infectious exposures by HCWs, thus reinforcing the need to report exposures and use personal protective equipment.

Furthermore, identification of problem areas in the workplace culture or barriers discouraging reporting need to be addressed. It is imperative to ensure reports are handled promptly and confidentially. Reporting procedures need to be covered in the curriculum in the initial and subsequent in-service training. Well maintained records of exposures are important tools to look for patterns of exposure and seek solutions to prevent future exposures as it allows the organisation to provide appropriate, prompt, medical assessment and treatment [10]. This has to be coupled with ensuring that HCWs are well acquainted with the infection control policies in place.

It is crucial to have a strategy that would establish standards, procedures and methods for HAIs surveillance, prevention and control and promote their implementation at organisational and national level [5]. Employers should have in place a system that includes written protocols for prompt reporting, evaluation, counselling, treatment, and follow-up of occupational exposures that may place a worker at risk of bloodborne pathogen infection [6]. HCW's education, HAIs surveillance, appropriate legislation and basic infection control measures are the essential elements of an infection control programme and the safety of health care workers is paramount to effective infectious disease mitigation or containment [3,4]. In the Armed Forces, interventions to improve UPs compliance amongst HCWs are urgently needed, as they are experiencing relatively high levels of $\mathrm{BBF}$ and NSIs and better compliance has been shown to be associated with fewer exposures [2].

\section{References}

1. World Health Report 2002: Reducing risks, promoting healthy life. Available from: http://www.who.int/whr/en. Accessed Jan 2009.

2. Sangwan BR, Kotwal A, Verma AK. Occupational exposure to blood and body fluids and risk of blood-borne virus infection amongst health care workers in a medical college hospital in Pune. MJAFI 2011; 67: 21-4.

3. Zimmerman Peta-Anne. Health care worker safety: preparing for the pandemic (Editorial). Newsletter of the Safe $\mathrm{H}$ and $\mathrm{S}$ network. 2009; 3: 5. (Available at http://www.uow.edu.au/health/ safehands/index.html). Accessed Nov 2010.

4. Lazzari S, Allegranzi B, Concia E. Making hospitals safer: the need for a global strategy for infection control in health care settings. World Hosp Health Serv 2004; 40: 40

5. Sepkowitz KA. Occupationally acquired infections in health care workers. Part I. Ann Intern Med 1996; 125: 826-34.

6. Beltrami EM, Williams IT, Shapiro CN, Chamberland ME. Risk and management of blood-borne infections in health care workers. Clin Microbiol Rev 2000; 13: 385-407.

7. Duseja A, Arora L, Masih B, Singh H, Gupta A, Behera D, Chawla YK, Dhiman RK. Hepatitis B and C virus - prevalence and prevention in health care workers. Trop Gastroenterol 2002; 23: $125-6$

8. Baffoy-Fayard N, Maugat S, Sapoval M, Cluzel P, Denys A, Sellier $\mathrm{N}$ et al. On behalf of the study group on hygiene practices in interventional radiology. Potential exposure to hepatitis $\mathrm{C}$ virus through accidental blood contact in interventional radiology. J Vasc Interv Radiol 2003; 14: 173-9.

9. Sencan I, Sahin I, Yildirim M, Yesildal N. Unrecognized abrasions and occupational exposures to blood-borne pathogens among health care workers in Turkey. Occup Med (Lond) 2004; 54 : 202-6.

10. First Responders: Encourage Your Workers to Report Bloodborne Pathogen Exposures. NIOSH Publication No. 2008118. Available at http://www.cdc.gov/niosh/docs/2008-118/ default.html. Accessed Dec 2010. 